\title{
Studies of the activated carbons used in double-layer supercapacitors
}

\author{
Deyang $\mathrm{Qu}^{*}$ \\ Emtech, 2486 Dunwin Dr., Mississauga, Ont., Canada
}

Received 24 January 2002; accepted 19 February 2002

\begin{abstract}
The specific capacitance of activated carbons is determined by both the ratio of edge/basal orientation and the nature of functional group on the surface. The difference between the edge and the basal layers results from the semiconductive properties of basal layer. The ratio of edge/ basal orientation can be estimated by X-ray diffraction (XRD). The wettability of activated carbons is determined by the nature of functional groups on the surface. Most of the surface groups are electrochemically active. The impact of the surface groups on electrochemical performance of the activated carbon electrodes was investigated by means of various surfactant treatments. The ac impedance and constant current discharge techniques were used. Two types of surface groups which had "capacitor-like" or "battery-like" behaviors, respectively, were revealed and discussed in detail. The surface groups with "battery-like" behavior should be avoided. "Non-symmetric" electrode arrangement should be considered for a double-layer supercapacitor in order to take the advantages of pseudo-capacitance of the surface groups with "capacitor-like" behaviors. (C) 2002 Elsevier Science B.V. All rights reserved.
\end{abstract}

Keywords: Supercapacitor; Surface groups; Pseudo-capacitance; Edge/basal layers; Double-layer; Activated carbon

\section{Introduction}

Non Helmholtz [1], Gouy [2], Chapman [3] and Stern [4] discovered that charges can be separated and stored at the interface between a conductor and a liquid electrolyte. Unlike a conventional capacitor, in strong and concentrated electrolyte solutions, the distance between the charges of the opposite sign across the electrode/electrolyte interface can be in the range of angstroms. Therefore, by choosing a suitable high surface area electrode material, a practical "supercapacitior" with specific capacitance of, e.g. $120 \mathrm{~F} / \mathrm{g}$ can be engineered.

In parallel with the double-layer types of "supercapacitor," Conway [5] discussed the transition from "supercapacitor" to "battery behavior" in electrochemical energy storage and the origin of "pseudo-capacitor" [6], which could be considered as "capacitor behavior." Much work has been reported in the area and reviewed [7].

Although the concept of storing relatively high electrical energy in reasonably small capacitors was proposed about three decades ago [8], the real efforts of research and

\footnotetext{
* Present address: Rayovac, 601 Rayovac Dr., Madison, WI 53719, USA. Tel.: +1-608-275-4745.

E-mail address: qu@rayovac.com (D. Qu).
}

development toward practical commercialization only happened over the past 10 years. The driving force behind the activities was increasing interest in the use of electrochemical systems under the conditions in which the electrical power demand is highly time dependent, often in short term pulses, e.g. electrical vehicles, digital communication devices, digital cameras, etc.

Theoretically, in order to achieve high capacitance, high surface area carbon should be chosen as electrode material. However, activated carbon manufactured under different conditions may have different porosity and surface conditions, so not all the BET surface area is electrochemically accessible. For those accessible surface areas, the specific capacitance $\left(\mathrm{mF} / \mathrm{cm}^{2}\right)$ may not be the same. In this paper, the impact of the structure and the surface condition of carbon will be discussed in detail.

\section{Experimental details}

\subsection{Material}

The activated carbons were either made or purchased from SPECTRACAB. All carbons used in the structure investigation were reflux washed with acetone in a Soxhlet 
extractor for $48 \mathrm{~h}$ to remove most of the weakly bonded surface functional groups inherited from their pre cursors and the manufacturing processes. Teflon suspension (T-30) used was from Dupont.

\subsection{Electrolyte, reference and counter electrodes}

An amount of $30 \%$ aqueous potassium hydroxide solution was used as electrolyte in all experiments at $298 \pm 1 \mathrm{~K}$. All potentials reported, unless otherwise specified as the voltage of the two electrode systems, were referred to the $\mathrm{Hg} / \mathrm{HgO}$ reference electrode immersed in $\mathrm{KOH}$ of the same concentration as the experimental electrolyte. A Ni-mesh counter electrode was used.

\subsection{Construction of electrodes used in the experiments}

Electrodes were punched out of activated carbon sheet and the discs were then mounted onto a $\mathrm{Ni}$-current collector in a Teflon cell holder by means of screw-fitting plug. If a two-electrode system was used, two disc electrodes of the same size and same type (from the same carbon sheet) were mounted face to face into the holder with a non-woven separator in between. Before the electrochemical measurements were performed, all electrodes were vacuum wetted.

In the event of the surfactant treatments, the disc electrode was soaked in the diluted surfactant solution for $10 \mathrm{~h}$, then the electrode air dried before mounting in the cell holder for the electrochemical testing.

\subsection{Experimental techniques and instrumental details}

The ac impedance measurements were conducted by means of a Solartron Electrochemical Interface 1278 and Solartron FRA 1255 controlled by ZPLOT. The equivalent circuit fitting of the ac impedance data was done using
ZVIEW. Constant current charge and discharge were carried out by means of HOKUTO Denko HA 301 potentiostat/ galvanostat controlled by a Q\&R Smart Date package.

The X-ray powder diffraction pattern of each sample was collected using a Siemens D5000 powder diffractometer equipped with a $\mathrm{Cu}$ target $\mathrm{X}$-ray tube and monochrometer.

\section{Results and discussion}

\subsection{The effect of carbon structure}

\subsubsection{The structure of activated carbons}

Activated carbons are high surface area, high porosity carbons that are produced by either thermal activation or chemical activation. The carbons are made of small hexagonal carbon rings, which are called "graphene sheets." The size orientation and stacking of these sheets is determined by carbon preparation method. Generally, there is very little order between the sheets and no long range threedimensional order.

Dahn and co-workers proposed the model of "falling cards" to explain the complex structure of activated carbon [9]. During the activation processes of sample preparation, thermal energy may be sufficient to break the links between adjacent graphene sheets, allowing some to rotate into parallel orientation. So, in that instance, an activated carbon can be treated as the combination of a lot of small domains that consist of a few graphene sheets in parallel. The lateral extent of the graphene layers $\left(L_{\mathrm{a}}\right)$ can be estimated from the width of $\left(\begin{array}{lll}1 & 1 & 0\end{array}\right)$ and $\left(\begin{array}{lll}1 & 0 & 0\end{array}\right)$ X-ray diffraction (XRD) peaks while the stacking number of graphene sheets $\left(L_{\mathrm{c}}\right.$, or $\left.N\right)$ can be estimated through the width of $\left(\begin{array}{ll}0 & 0\end{array}\right)$ peak. By knowing the $L_{\mathrm{a}}$ and $L_{\mathrm{c}}$, the size of regions of organized carbon that is in a roughly parallel fashion can be given. Fig. 1 shows a typical powder XRD patterns for the activated carbons

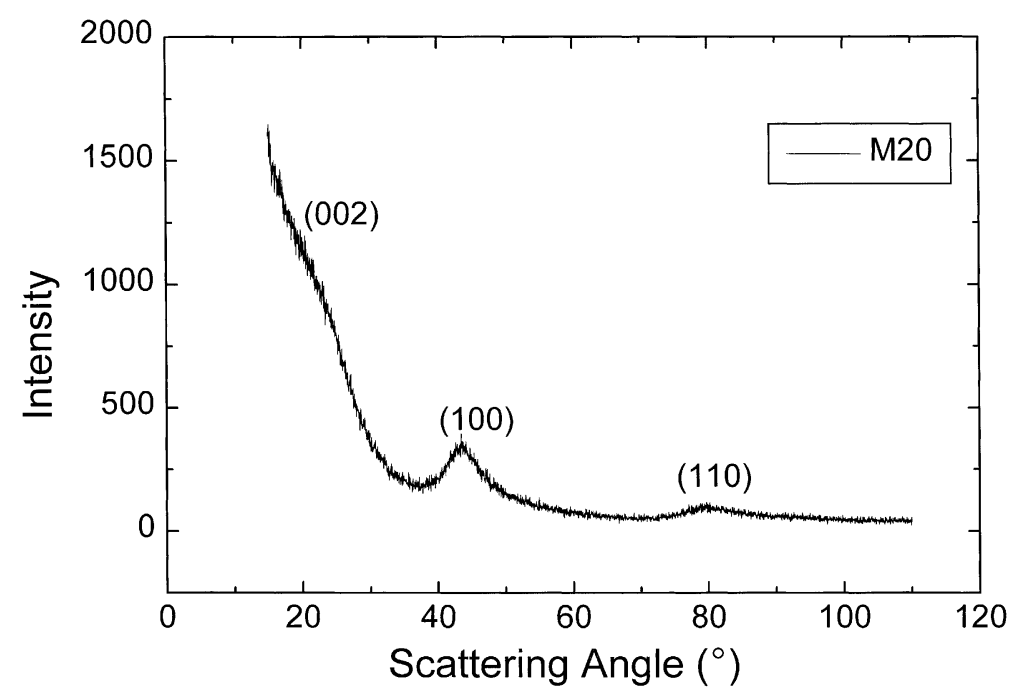

Fig. 1. XRE profile of activated carbon M-20. 
studied in this paper. The clear observation of $\left(\begin{array}{lll}1 & 0 & 0\end{array}\right)$ peak near $42^{\circ}$ and $\left(\begin{array}{lll}1 & 1 & 0\end{array}\right)$ peak near $79^{\circ}$ indicating that the activated carbon consists of small domains of ordered graphene sheets. In order to examine the stacking of the layers, Liu et al. [9] calculated theoretical (l 002 ) Bragg peaks for powder samples made up of randomly oriented single-layers, bilayers and trilayers, as well as the mixture of randomly oriented bilayers. According to Liu's results, all activated carbons studies in this paper should contain randomly oriented single- and multi-layer domains.

\subsubsection{Double-layer capacitance on the basal and edge orientations of graphite}

A significant difference is found in the double-layer capacitance for the basal and the edge orientation of graphite. The double-layer capacitance for the edge orientation is an order of magnitude higher than that on the basal layer. Kinoshita [10] tabulated a series of values of the differential capacitance for various graphite materials. The low capacitance for the basal layer is attributed to the semiconductor properties of graphite along the direction of basal orientation. Randin and Yeager [11-13] carried out detailed investigation for the differential capacitance on the surface of both edge and basal plane of stressed annealed pyrolytic graphite.

The anisotropic electric properties of graphite result from its crystal structure. The ratio of principal axis ( $a$ - and $c$-axis) conductivities of single crystal graphite is higher than that of other element material. Along the direction of edge orientation, graphite can be treated as conductor-like metal. However, it shows the properties of semiconductor along the direction perpendicular to the graphene layers. The capacitance versus potential curves for basal orientation [11] was symmetrical V-shaped curves with minima at $0.0 \mathrm{~V}$ versus NHE and independent of the chemical identity of the electrolyte. So, the graphite in the direction normal to the basal layer can be viewed as an intrinsic semiconductor.

Fig. 2 shows the potential distribution at the basal layer of graphite under negative bias potential. The differential capacitance of a semiconductor interface is composed of three series components: the capacitance of the space charge layer within the semiconductor, that of the compact doublelayer and that of the diffuse ionic layer of the electrolyte. As an intrinsic semiconductor, the charge-carrier (hole and electron) densities are very low and similar to the solution with very low electrolyte concentration. Consequently, the charge carriers distribute away from the interface and extend into the bulk of the graphite over a large distance, which is inversely related to the charge carrier density. The charge distribution distance inside the basal orientation of graphite is of the same order of magnitude as that the of diffusion layer in very dilute solutions, the mathematical treatment for the space charge region inside semiconductor is identical to that for the diffusion layer on the solution side. Therefore, the differential capacitance $(C)$ of graphite at its basal layer

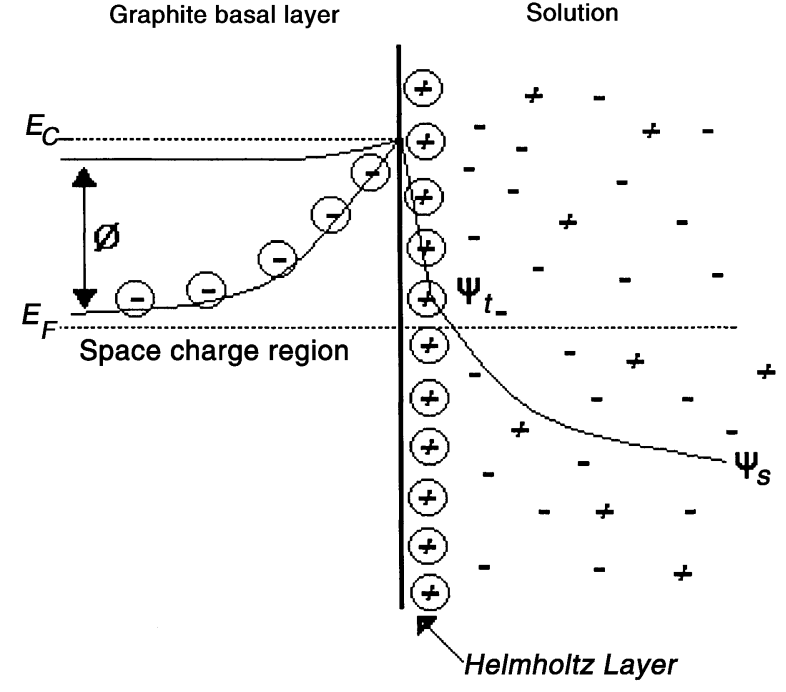

Fig. 2. Potential profile at the graphite basal layer/electrolyte interface under negative bias potential. $E_{\mathrm{c}}$ and $E_{\mathrm{F}}$ are potentials of conductive band and Fermi-level, respectively.

can be represented as

$\frac{1}{C}=\frac{1}{C_{\mathrm{SC}}}+\frac{1}{C_{\mathrm{H}}}+\frac{1}{C_{\mathrm{diff}}}$

where $C_{\mathrm{SC}}$ is the capacitance of the space charge region inside basal graphite, $C_{\mathrm{H}}$ the Helmholtz double-layer capacitance, and $C_{\text {diff }}$ is the capacitance for the diffusion doublelayer. For concentrated electrolyte solutions, e.g. $9 \mathrm{~N} \mathrm{KOH}$ solution for alkaline supercapacitors, $C_{\text {diff }}$ and $C_{\mathrm{H}}$ are high, compared to $C_{\mathrm{SC}}$ since the basal graphite is an intrinsic semiconductor with very low charge-carrier density. Therefore, their contributions can be ignored. As a consequence, the low capacitance at the basal layer of graphite is best attributed to the space charge component within the graphite. In addition, bias potential on the basal graphite mainly changes the potential of the space charge region and has little impact on the potential in the Helmholtz layer.

For the edge orientation of graphite, one can treat it the same way as a metal electrode, since the charge carrier density or conductivity of edge graphite is at the same level as a metal. The charges inside the graphite are limited within a few angstroms of the interface.

\subsubsection{Specific double-layer capacitance for activated carbons}

In the light of the discussion in Sections 3.1.1 and 3.1.2, an activated carbon can be treated as a mixture of microdomains of ordering graphene sheets. The average $L_{\mathrm{a}}$ and $L_{\mathrm{c}}$ are determined by the raw material and the manufacture procedure. The specific double-layer capacitance $\left(\mathrm{F} / \mathrm{cm}^{2}\right)$ of an activated carbon is the sum of the capacitance on the surface of each micro-domains. It can be divided into two categories: the capacitance on the basal orientation of the domains and the capacitance on the edge orientation of the domains. Therefore, the specific capacitance of an activated 


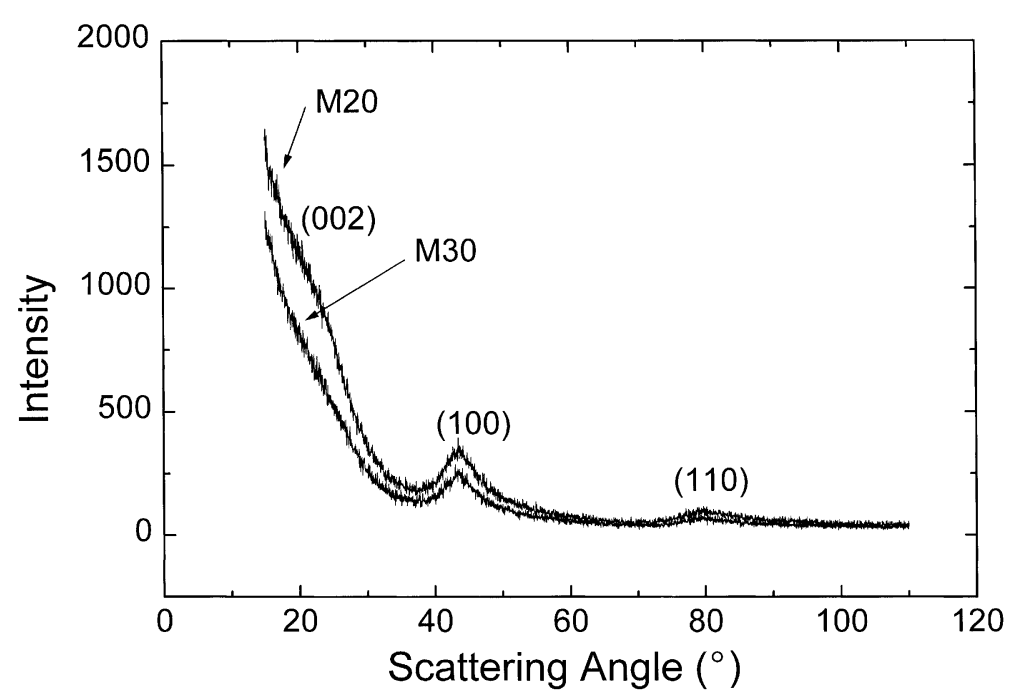

Fig. 3. Comparison of XRD profiles of activated carbons M-20 and M-30.

carbon can be assumed to be proportional to the aspect ratio $\left(L_{\mathrm{c}} / L_{\mathrm{a}}\right)$.

All activated carbons were Flex washed with acetone to remove the weakly bonded surface groups. The capacitance of the activated carbons was obtained through an ac impedance technique at $0.01 \mathrm{~Hz}$.

Fig. 3 shows the comparison of XRD spectrum for M-20 and M-30. Both carbons can be treated as a mixture of randomly orientated single graphene layer and bilayer domains [9]. The peak height of XRD can be considered proportional to the crystal size, e.g. $\left(\begin{array}{lll}0 & 0 & 2\end{array}\right)$ peak height is proportional to $L_{\mathrm{c}}$ and $\left(\begin{array}{lll}1 & 0 & 0\end{array}\right)$ peak height is proportional to $L_{\mathrm{a}} . L_{\mathrm{c}}$ for both M-20 and M-30 are similar, while $L_{\mathrm{a}}$ size for M-20 is smaller than that of M-30. So, the aspect ratio for M-20 was larger than that of M-30, therefore, M-20 had higher specific capacitance than M-30 (Table 1).

EU-1 to EU-4 activated carbons were homemade from the same precursor through different carbonization and activation processes. So, $L_{\mathrm{a}}$ for all four carbons were similar, while the $L_{\mathrm{c}}$ value varied. Fig. 4A shows the XRD of the activated carbons. The large sharp peak resulted from metal impurity inherited from the precursor. The straight lines show the background subtracted. Fig. 4B shows the diffraction peaks with background subtracted. The heights of $\left(\begin{array}{lll}0 & 0 & 2\end{array}\right)$ peaks are listed in Table 1. The $L_{\mathrm{a}}$ values for all four carbons were calculated using the Scherrer equation [14] and are also tabulated in
Table 1. Obviously, for the group of four carbons, $L_{\mathrm{a}}$ values are similar, while $L_{\mathrm{c}}$ values varies a lot. The specific capacitance for the carbon increased as $L_{\mathrm{c}}$ increased.

In summary, the specific capacitance of an activated carbon relies on the crystal orientation on the surface. The carbons with a higher percentage of edge orientation on the surface have higher specific capacitance. However, together with the crystal orientation on the surface, one sees the significance of the specific surface area. The gravimetric capacitance of an activated carbon is: surface area $\left(\mathrm{m}^{2} / \mathrm{g}\right) \times$ specific capacitance $\left(\mathrm{F} / \mathrm{m}^{2}\right)$.

In general, the activated carbons suitable for the supercapacitor application have a surface area larger than $2000 \mathrm{~m}^{2} / \mathrm{g}$. Those activated carbons, e.g. M-20, M-30 and EU-4 in Table 1, which were fully activated, can be considered as a mixture of randomly oriented single layers and bilayer domains. Therefore, choosing precursors and proper carbonization and activation processes, which can result of lower $L_{\mathrm{a}}$ value, would be the key of making the activated carbons with high specific capacitance.

\subsection{The effect of surface functional groups}

An ideal electrochemical double-layer capacitor can be represented by a capacitor in series with a resistor. This can only be demonstrated experimentally, under strict environmental

Table 1

Comparison of specific capacitance, surface area and crystal size

\begin{tabular}{lllll}
\hline & BET surface $\left(\mathrm{m}^{2} / \mathrm{g}\right)$ & Capacitance $\left(\mathrm{F} / \mathrm{cm}^{2}\right)$ & $L_{\mathrm{a}}(\AA)$ & Peak height $(002)$ \\
\hline M-20 & 2100 & 0.106 & 10.1 & Bilayer \\
M-30 & 2571 & 0.0282 & 14.5 & Bilayer \\
EU-1 & 1294 & 0.0554 & 15.5 & 580 \\
EU-2 & 1156 & 0.0458 & 16.0 & 400 \\
EU-3 & 1450 & 0.0340 & 15.8 & 250 \\
EU-4 & 2440 & 0.0215 & 16.2 & 100 \\
\hline
\end{tabular}



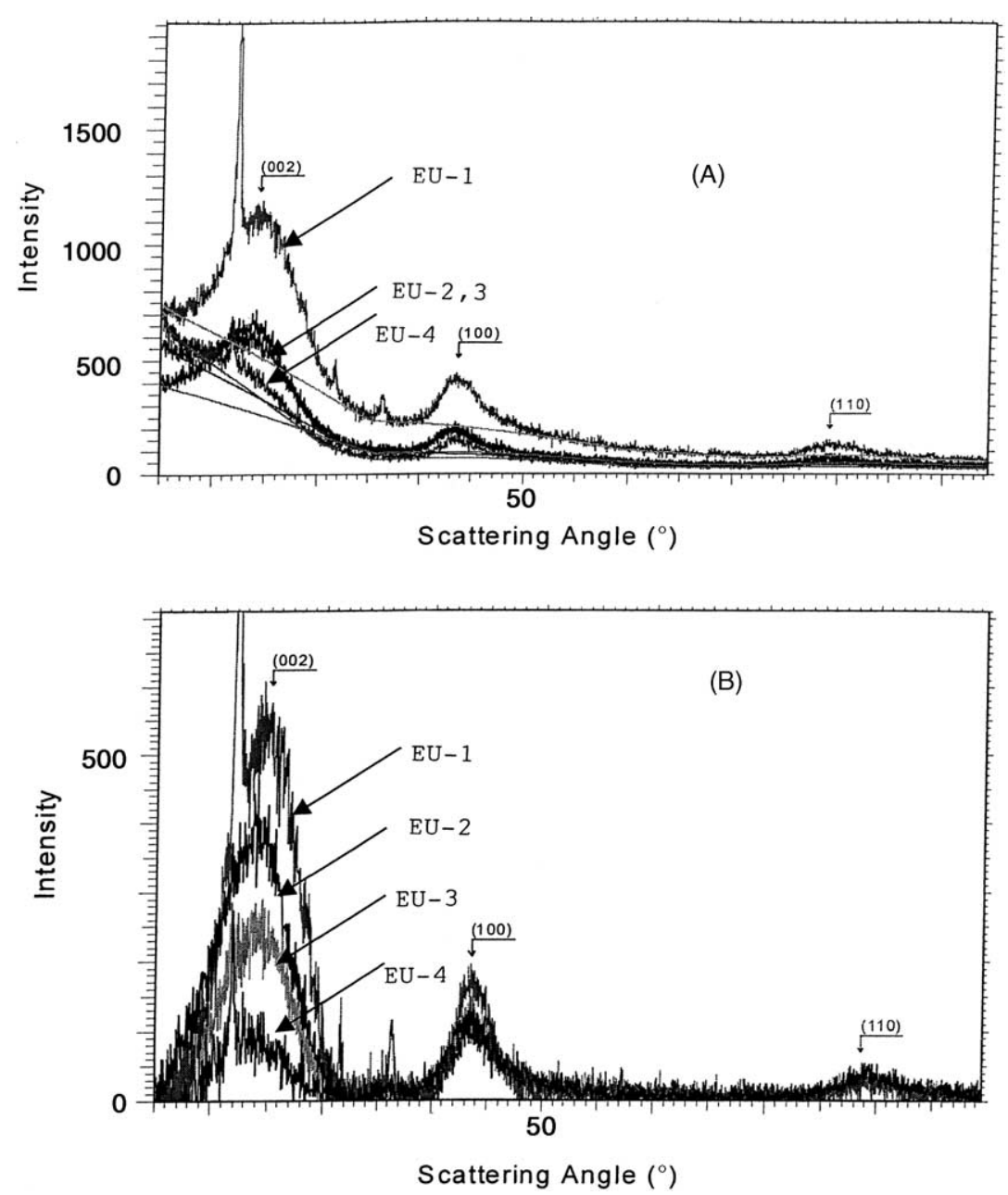

Fig. 4. Comparison of XRD profiles of activated carbon EU-1 to EU-4, straight lines show how background being subtracted (A), XRD profile of EU-1 to EU-4 with background subtracted (B).

control, for well-cleaned electrodes, for instance, Hg electrode in acid solution or Au electrode in alkaline. Practically, porous electrodes used in double-layer capacitors contain pores of various sizes, the micro-porosity of the carbon provides high surface area and contributes in a major way to the double-layer capacitance. However, the same porous structure makes the electrodes more complex, not only due to the kinetics of electrochemical accessibility to the micropores [15], but also owing to the presence of various surface functional groups. Those surface groups could include acidic or basic functionalities such as quinoid, quinhydrone, phenolic, carboxyl, carbonyl and lactone. Additionally, under special conditions, sulfate surface compounds, hydrogen-bonded interlayer groupings and free radical may also be present.

The basal plane, as discussed in the previous section, is flat on the atomic scale and can be considered as a nearly perfect single crystal. Because there are no unsatisfied chemical bonds or free electrons, it is unlikely for functional groups to be chemically bonded to its surface, except to the defect sites with high strains. The edge plane is rough and its electrochemical properties differ considerably from those of the basal layer. Although, the low capacitance obtained from the basal plane is attributed to its semiconducting properties, the higher capacitance for the edge layer is at least partially attributed to the contribution of the functional groups on the surface. As the reactivity of carbon atoms with unsatisfied valences on edge sites is greater than that of carbon atoms in the basal plane, the chemical properties of carbon vary with the relative fraction of edge sites and basal sites on the surface.

In the case of redox surface functionalities, which reside at the interface of carbon particles (mainly at the edge plane), their relative proportion in the material tends to scale with the specific surface area of the material and the fraction of edge layers. For such redox reactivity, a significant redox pseudocapacitance, $C_{\phi}$ arises as well as the desired predominant double-layer capacity, $C_{\mathrm{dl}}$. Besides the contribution of psuedocapacitance, some functional groups on the surface can influence the wettability of carbon material, it is considerable important in maximizing the access of electrolyte to the surface of porous carbon electrode. The influence of surface 
functional groups on the double-layer capacitance can be summarized in the follows: electrolyte and the contact between the electrode and the current collector, $C_{\phi}$ stands for pseudo-capacitance through a

functional groups $\left\{\begin{array}{l}\text { no electrochemial reactions }\left\{\begin{array}{l}\text { more hydrophilic } \\ \text { more hydrophobic }\end{array}\right. \\ \text { with electrochemial reactions }\left\{\begin{array}{l}\text { irreversible reactions }\left\{\begin{array}{l}\text { more hydrophilic } \\ \text { more hydrophobic }\end{array}\right. \\ \text { reversible reactions }\left\{\begin{array}{l}\text { pseudo-capacitance behaviors } \\ \text { battery behaviors }\end{array}\right.\end{array}\right.\end{array}\right.$

Functional groups, which are electrochemically inert in the potential range of operation, will either enhance the wettability of the activated carbon electrodes, thus, the specific capacitance of the carbon increases due to the more efficient surface utilization; or make the surface of carbon less wettable and hydrophobic, thus, the specific capacitance decreases. More complex situations arise for those functional groups, which are themselves reduced or oxidized in the potential range of the operation of the supercapacitor. The characteristics of functional groups which experience irreversible redox reaction can be permanently changed. They could become either more hydrophobic, which would have positive impact on the performance of electrodes, or more hydrophilic with negative impact.

\subsubsection{Surface groups with pseudo-capacitance behaviors}

As mentioned previously, pseudo-capacitance arises when the potential at which a charge is being passed is a function of the integrated charge accumulated, rather than function of log of current density (Tafel relation), due to special thermodynamic reasons. Functional groups absorbed on the surface of a porous electrode could be oxidized or reduced with the electrode potential. In the case of the high surface area activated carbon electrodes, the pseudo-capacitance associated with redox reactions of surface functional groups is always coupled with the non-Faradaic double-layer capacitance. The specific capacitance will be influenced by those functional groups. Fig. 5 shows than equivalent circuit to represent the situation. $R_{\mathrm{S}}$ is the Ohmic resistance from the

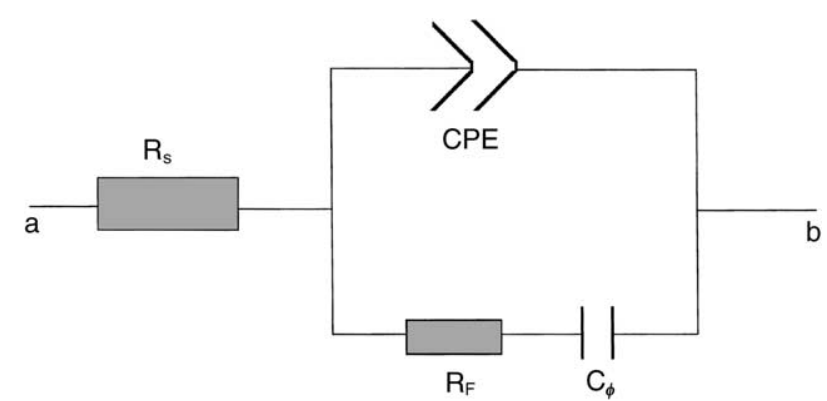

Fig. 5. Equivalent circuit model used to fit the ac impedance data. $R_{\mathrm{s}}$, Ohmic resistance; $R_{\mathrm{F}}$, Faraday resistance; $C$, pseudo-capacitance; CPE, constant phase element impedance.
Faradaic resistance $R_{\mathrm{F}}$, while $\mathrm{CPE}$ is the constant phase element impedance.

In the case in which ac signal penetration depth is comparable to the depth of the pores, the porous electrode can be treated as a $R C$ transmission line network. The mathematical equation of the transmission line has the same form as the diffusion equation since the process taking place in an $R C$ circuit may be treated as electronic diffusion into a semiinfinite medium. Its impedance can be represented as

$Z=\sigma^{\prime} \omega^{-m}\left[\cos \left(\frac{1}{2} m \pi\right)-\mathrm{i} \sin \left(\frac{1}{2} m \pi\right)\right], \quad 0<m<1$

where $\sigma^{\prime}$ is termed as the constant phase element (CPE) factor, $m$ is the CPE exponent.

$$
\begin{aligned}
& R^{\prime}=\sigma^{\prime} \omega^{-m} \cos \left(\frac{1}{2} m \pi\right) \\
& C^{\prime}=\frac{1}{\sigma^{\prime} \omega^{1-m} \sin (1 / 2) m \pi}
\end{aligned}
$$

where $R^{\prime}$ and $C^{\prime}$ represent the distributed resistance and the distributed double-layer capacitance of the porous electrode at a given modulation frequency.

Activated carbon E-10 was treated by a sulflonate surfactant. A surfactant is a material that, when dissolved in a solvent, concentrates at liquid/solid interface. In the case of porous activated carbon materials, the surfactants coat the pore walls, a surfactant can make the surface "active," because half of the molecular is hydrophobic to the liquid that it is dissolved in and will be attached to the surface of the solid, while another half of the molecular is hydrophilic, which can make the solid surface more wettable.

Evidently, the impact of surfactant on the wettability of an activated carbon electrode can be easily observed. The nontreated activated carbon electrode floats on the surface of $\mathrm{KOH}$ electrolyte; the same electrode, treated with the surfactant absorbs the electrolyte and sinks to the bottom of the container, gas bubbles can be seen rising from the electrode. Any surfactants can make an electrode more wettable, but electrochemically active functional groups could be introduced on to the surface of the electrode during the treatment. The potential of the carbon electrode varies continuously during he charge/discharge processes. The electrochemically active surface groups could react at a certain potential or potential ranges. Due to the complexity of the organic compounds, the types of electrochemical reactions taking place in 


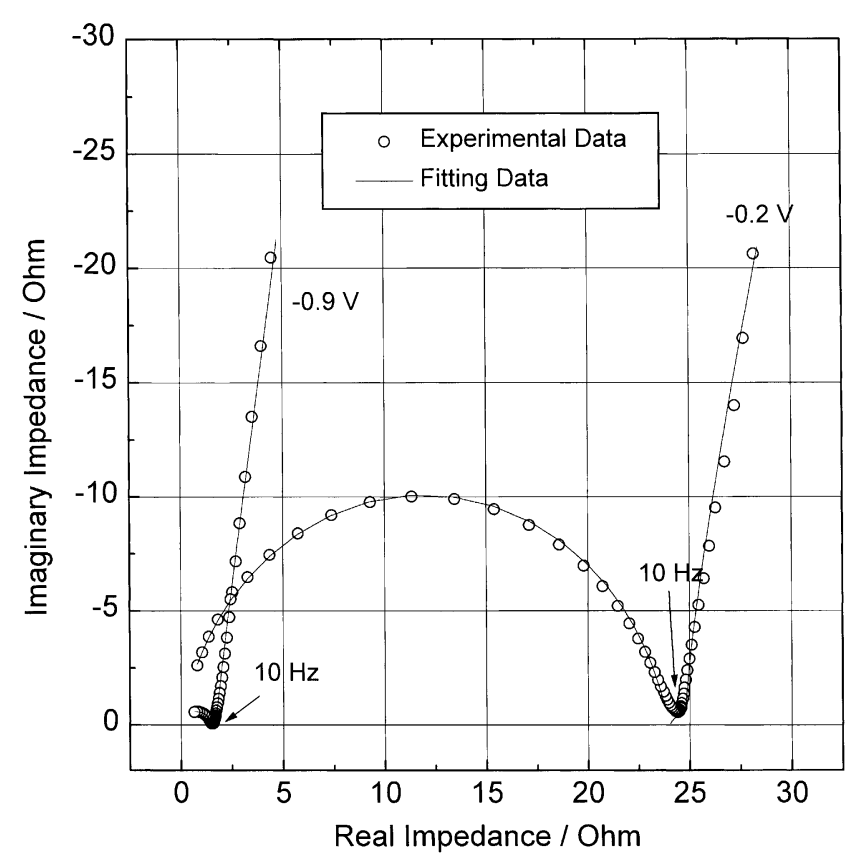

Fig. 6. Comparison of ac impedance responses for E-10 activated carbon electrode treated with sulflonate surfactant at -0.2 and $-0.9 \mathrm{~V}$. Numerical fitting was based on the equivalent circuit shown in Fig. 5. Three-electrode system was used, potential was against $\mathrm{Hg} / \mathrm{HgO}$ reference electrode.

the different potential ranges may be different, thus, the apparent capacitance of an electrode within different potential ranges could be different. As far as those Faradic reactions can meet the criteria of "pseudo-capacitance" [5], they would have a positive impact to the "apparent capacitance".

Fig. 6 shows the ac impedance spectrum for the activated carbon cloth electrode (E-10) treated with a sulfonate surfactant. The impedance was measured at -0.2 and $-0.9 \mathrm{~V}$ versus $\mathrm{Hg} / \mathrm{HgO}$ reference electrode within the frequency range of $100 \mathrm{kHz}-10 \mathrm{mHz}$. The impedance can only be well fitted separately for the frequency ranges $10 \mathrm{mHz}-10 \mathrm{~Hz}$ and $10-100 \mathrm{kHz}$. This phenomena could result from the fact that surfactant polymer can only coat the wall of pores of large size; owing to the large molecule size, the surfactant may not enter the pores with small openings. Therefore, at high frequency range, rather than the double-layer, Faradaic reaction of the organic surfactant was the major contributor to the overall impedance, since only small portions of the electrode surface area was accessed in the frequency range.
The impedance measured at both potentials was fitted based on the equivalent circuit shown in Fig. 5. Table 2 tabulates the fitting results.

It is clear that the Faradaic reaction for the surfactant at $-0.9 \mathrm{~V}$ was not the same as that at $-0.2 \mathrm{~V}$. Fig. 7 shows the first a few "charge" or "discharge" curves of the same electrode as that in Fig. 6. A three-electrode system was used, the potential of the activate carbon electrode was measured against $\mathrm{Hg} / \mathrm{HgO}$ reference electrode. Two regions with different slopes can be distinguished. The apparent capacitance for the region of -0.5 to $-1.0 \mathrm{~V}$ is $7.5 \mathrm{~F}$, while the apparent capacitance for the region of -0.5 to $0.2 \mathrm{~V}$ is $5.5 \mathrm{~F}$. Total capacitance at -0.9 and $-0.2 \mathrm{~V}$ was calculated from ac impedance results at low frequency $(0.01 \mathrm{~Hz})$, they were 7.2 and $5.1 \mathrm{~F}$, respectively. The "apparent capacitance" obtained from dc discharge were in good agreement with those obtained from ac impedance. It worth emphasizing that:

(1) The redox reaction a $-0.2 \mathrm{~V}$ was less reversible than that at $-0.9 \mathrm{~V}$, since at high frequency region, $R_{\mathrm{F}}$ at $-0.2 \mathrm{~V}$ was larger than that at $-0.9 \mathrm{~V}$. Thus, the electrode surface would be more permanently modified if it was cycled in a less negative potential range. Fig. 7 shows that the shift of "charge" curves for the -0.5 to $+0.2 \mathrm{~V}$ potential range are more pronounced during cycling than that for the -0.5 to $-0.9 \mathrm{~V}$ potential range.

(2) The surfactant at $-0.9 \mathrm{~V}$ oxidation state was much more wettable than that at $-0.2 \mathrm{~V}$. Double-layer capacitance at $-0.9 \mathrm{~V}$ is higher than that at $-0.2 \mathrm{~V}$. However, the surface group contributes more pseudocapacitance at -0.2 than $-0.9 \mathrm{~V}$.

An individual surfactant may favor the absorption of either anions or cations or both, due to electrostatic forces and specific chemical absorption. In Fig. 7, the process in -0.5 to 0.2 V actually includes displacing cations away from the electrode and accepting anions into the double-layer; the process in the range -0.5 to $-0.9 \mathrm{~V}$ included displacing anions and accumulating cations. The transformation from cation accumulation in the double-layer to the anion accumulation became more and more apparent during the cycling. It is interesting to point out that the apparent capacitance of the regions remained almost uncharged, since the charging curves of each region during cycling were almost parallel. Therefore, even though there may be some

Table 2

Fitting results based on the equivalent circuit shown in Fig. 5

\begin{tabular}{|c|c|c|c|c|}
\hline Parameters & $-0.9 \mathrm{~V}(10 \mathrm{mHz}-10 \mathrm{~Hz})$ & $-0.9 \mathrm{~V}(10-100 \mathrm{kHz})$ & $-0.2 \mathrm{~V}(10 \mathrm{mHz}-10 \mathrm{~Hz})$ & $-0.2 \mathrm{~V}(10-100 \mathrm{kHz})$ \\
\hline Charge transfer resistance $\left(R_{\mathrm{F}}\right)$ & 1.612 & 1.474 & 1.322 & 2.386 \\
\hline Pseudo-capacitance $\left(C_{\phi}\right)$ & 1.596 & 0.3147 & 2.672 & 0.2749 \\
\hline CPE factor $\left(\sigma^{\prime}\right)$ & 0.315 & 223.5 & 1.623 & 289.5 \\
\hline CPE exponent (m) & 0.88 & 0.85 & 0.7305 & 0.8937 \\
\hline $\begin{array}{c}\text { Double-layer capacitance at } \\
\text { the frequency of } 0.01 \mathrm{~Hz}\end{array}$ & 5.6 & & 2.5 & \\
\hline Total capacitance & 7.2 & & 5.2 & \\
\hline Apparent capacitance $(\mathrm{dc})$ & 7.5 & 5.5 & 5.5 & 7.5 \\
\hline
\end{tabular}




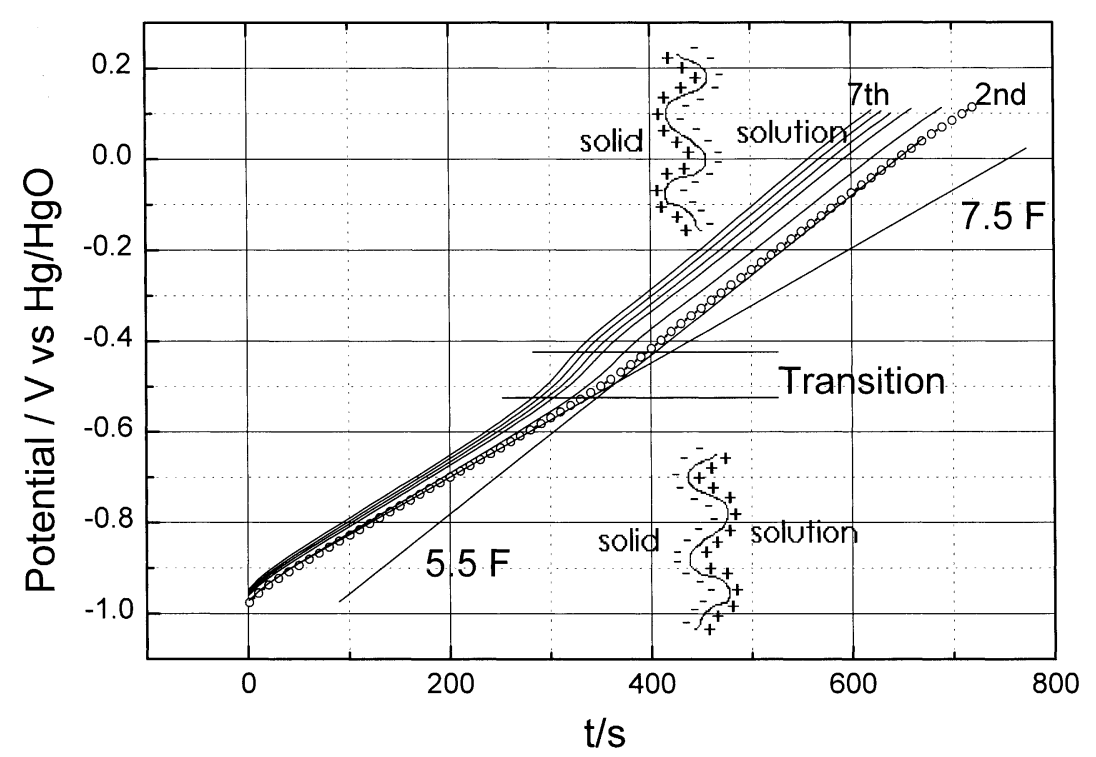

Fig. 7. The first seven "charge" curves for E-10 activated carbon treated with sulflonate surfactant. Transition of charge accumulation is shown. Cations were accumulated in -1.0 to $-0.5 \mathrm{~V}$, while anions were accumulated in -0.5 to $+0.2 \mathrm{~V}$. Three-electrode system was used, potential was against $\mathrm{Hg} / \mathrm{HgO}$ reference electrode.

permanent modifications during cycling, both Faradaic reactions were reversible.

\subsubsection{Surface functional groups with "battery-like" behavior}

Most electrochemical reactions follow the Tafel type of relation:

$i(\eta)=i_{0} \mathrm{e}^{-\alpha \eta \eta / R T}$

or

$\eta=a+b \ln i$

For these reactions, during the course of constant current discharge, the potential is, theoretically, constant and does not depend on the state of charge, e.g. $\mathrm{PbO}_{2} \rightarrow \mathrm{Pb}$, $\mathrm{NiOOH} \rightarrow \mathrm{Ni}(\mathrm{OH})_{2}, \mathrm{CdO} \rightarrow \mathrm{Cd}, \mathrm{ZnO} \rightarrow \mathrm{Zn}$, etc. However, in a practical battery system, when chemically well defined species are the battery reagents, they may not give unique single potentials on discharge, this usually results from the changing of interparticle contact and resistance, especially for those reactions with volume and phase charges.

The discharge process of an activated carbon electrode with surface functional groups having "battery-like" behavior is shown in Fig. 8. The electrode was made of E-10 activated carbon treated with a fluoro-surfactant. The third, fourth and fifth discharge curves are shown. In the region I of the discharge curves, the charges were from the discharge of the double-layer, "capacitor-like" curves are shown. When the discharge potential reached -0.65 to $-0.70 \mathrm{~V}$ versus $\mathrm{Hg} / \mathrm{HgO}$, which was the potential for the electrochemical redox reaction to take place, the discharge curve (region II) became flat. The charges resulted from converting

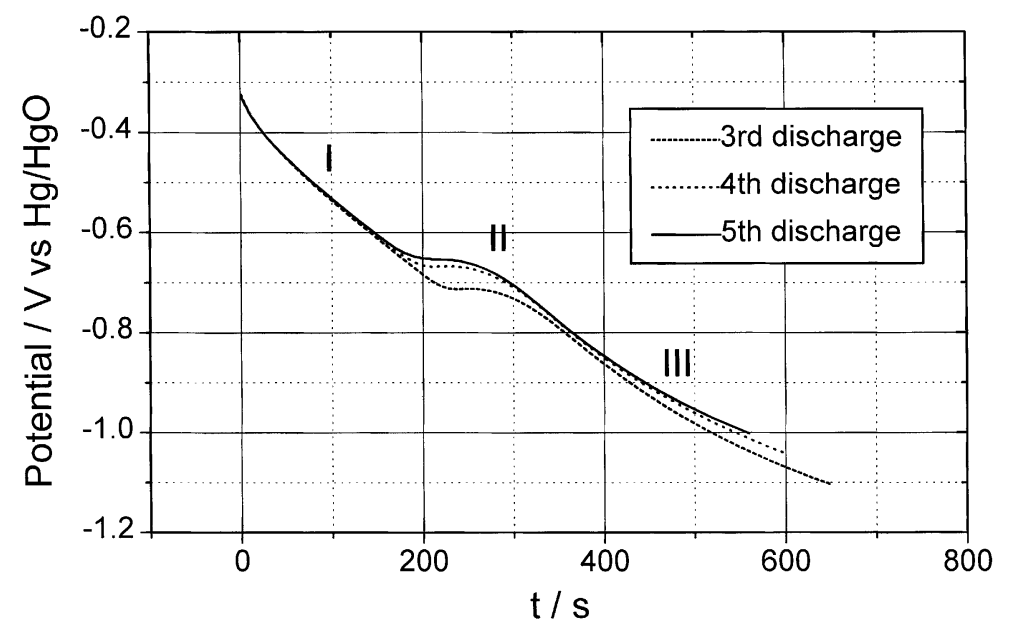

Fig. 8. The third, fourth, and fifth "discharge" curves for activated carbon E-10 treated with fluoro-surfactant. 
the oxidation states of the functional groups rather than from the double-layer. Apparently, the Faradaic reaction was reversible. After the redox reaction of the surface groups ended, the remaining double-layer charges started to relax again, this process was associated with the region III in Fig. 8. Although the slope of the discharge curve in region III may not necessarily be the same as that in region I, owing to the difference of oxidation state of the surface functional groups, the redox reaction in -0.65 to $-0.7 \mathrm{~V}$ did not affect the double-layer capacitance of the electrode, because the slope of the discharge curves before and after the reaction remained unchanged.

\subsubsection{Selections of modified activated carbon material for supercapacitor electrodes}

Selecting an activated carbon electrode with high specific capacitance is one of the major tasks in the development of supercapacitors. Surface modification is believed to be an effective means to achieve the goal. A properly modified activated carbon can become more wettable, allowing electrolyte to penetrate into small pores more rapidly; the surface functional groups may react reversibly and behave like a "pseudo-capacitor," therefore, the total "apparent" capacitance would increase. The potentials of the redox reactions are determined by the types of surface groups. Thus, the value of apparent capacitance of an electrode may be sensitive to the potential range.

In order to take advantage of the pseudo-capacitance associated with the redox reactions of surface functional groups, the electrochemical reactions should have small $R_{\mathrm{F}}$ values or lower activation energies for the charge transfer.

A supercapacitor assembly includes two electrodes which may either be fabricated from the same or different materials. In the instance in which the electrodes are fabricated of the same material, they are referred to as "symmetric electrodes." Conversely, if they are made from different materials, they are referred to as "asymmetric electrodes." During the charge and discharge of a supercapacitor, one electrode only accepts/releases anions $(+)$ while another electrode only accumulates/disposes cations ( - ). Activated carbons which have the same "apparent capacitance" throughout the whole operating potential range, e.g. bare E-10 activated carbon, are suitable for "symmetric electrode" supercapacitors. Activated carbon electrodes, which have different "apparent capacitance," can only be used in non-symmetric electrode systems. The activated carbon which favors anion "absorption" should be chosen for the positive electrode and the activated carbon which favors cation "absorption" for the negative electrode of a supercapacitor.

\section{Conclusions}

The influences of activated carbon structure and surface groups on the performance of a supercapacitor have been elucidated. The following points are examined noted:

1. The specific capacitance of an activated carbon relies on the crystal orientation of its surface. The higher percentage of edge orientation results of the higher double-layer capacitance and favors the stronger bonding of surface functional groups.

2. The difference between edge and basal orientation results from the semiconductor behavior of the basal layer and the unsatisfied chemical bonds on the edge layer.

3. The roles of surface groups in the carbon electrodes are: (i) the influence on the wettablity of the electrodes; (ii) the contribution of pseudo-capacitance to the apparent capacitance of the electrodes.

4. Surface functional groups may be electrochemically reactive in certain potential ranges.

5. The electrochemical behavior of surface groups can be "capacitor-like" or "battery-like".

6. Understanding the electrochemical performance of the materials over different potential ranges is key to the selection of positive electrode materials and negative electrode materials for "non-symmetric" supercapacitor systems.

7. Certain types of surfactants may favor either cation or anion absorption or both on the surface of electrode. The wettability of electrode may be influenced by the oxidation state of the surfactant.

\section{References}

[1] H. Non Helmholtz, Ann. Phys. 29 (1879) 337.

[2] G. Gouy, J. Phys. 9 (1910) 457.

[3] D.L. Chapman, Phil. Mag. 25 (1913) 475.

[4] O. Stern, Z. Elektochem. 30 (1913) 475.

[5] B.E. Conway, J. Electrochem. Soc. 30 (1924) 508.

[6] B.E. Conway, V. Birss, J. Wojtowice, J. Power Sources 1 (1997) 6.

[7] B.E. Conway, Electrochemical Supercapacitor, Kluwer Academic Publishers/Plenum Press, New York, 1999.

[8] H.E. Becker, General Electric, US Patent 2,800,616.

[9] Y. Liu, J.X. Xue, T. Zheng, J.R. Dahn, Carbon 32 (1996) 193.

[10] K. Kinoshita, Carbon, Wiley, New York, 1988.

[11] J.-P. Randin, E. Yeager, Electroanal. Chem. 36 (1972) 257.

[12] J.-P. Randin, E. Yeager, J. Electrochem. Soc. 118 (1971) 711.

[13] J.-P. Randin, E. Yeager, Electroanal. Chem. 58 (1975) 313

[14] B.E. Warren, X-ray Diffraction, Dover, New York, 1990.

[15] D. Qu, H. Shi, J. Power Sources 74 (1998) 99. 